\title{
Preoperative Embolization for Skull Base Meningiomas
}

\author{
Kensuke Suzuki ${ }^{1}$ Masaya Nagaishi ${ }^{1}$ Yoshiyuki Matsumoto ${ }^{1}$ Yoshiko Fujii ${ }^{1}$ Yuki Inoue ${ }^{1}$ \\ Yoshiki Sugiura $^{1}$ Koji Hirata ${ }^{1}$ Ryotaro Suzuki ${ }^{1}$ Yosuke Kawamura ${ }^{1}$ Ryuta Nakae ${ }^{1}$ Yoshihiro Tanaka ${ }^{1}$ \\ Akio Hyodo ${ }^{1}$ \\ ${ }^{1}$ Department of Neurosurgery, Dokkyo Medical University Koshigaya \\ Hospital, Koshigaya City, Saitama, Japan \\ J Neurol Surg B 2017;78:308-314. \\ Address for correspondence Kensuke Suzuki, MD, PhD, Department \\ of Neurosurgery, Dokkyo Medical University Koshigaya Hospital, 2-1- \\ 50, Minami-Koshigaya, Koshigaya City, Saitama 305-8555, Japan \\ (e-mail: kensukes@dokkyomed.ac.jp).
}

\begin{abstract}
Keywords

- meningioma

- skull base

- preoperative embolization

- endovascular surgery

- n-butyl cyanoacrylate

The results of preoperative embolization for skull base meningiomas were retrospectively evaluated to confirm the efficacy of this procedure. Skull base meningiomas that were treated with preoperative embolization were evaluated in 20 patients. The occluded arteries, embolic materials, treatment time, excision rate, neurologic manifestations, and complications were analyzed. The embolic material was $80 \%$ liquid, $30 \%$ coils, and $15 \%$ particles. The surgery was normally completed within 3 to 5 hours. Blood loss was normally approximately $250 \mathrm{~mL}$, excluding four patients having the following conditions: malignant meningioma, a large tumor located on the medial side of the sphenoidal ridge, the petroclival tumor, and infiltrated tumor into the sigmoid sinus. The mean excision rate was $90 \%$, achieving a Simpson grade III, but $10 \%$ were graded as Simpson grade IV. No permanent complications due to the preoperative embolization occurred. No neurologic symptoms occurred after excision. Current cerebral endovascular treatment is sophisticated, and the complication rate has markedly decreased. Although it was impossible to compare directly with or without operative embolization, preoperative embolization should be actively used as part of the treatment for this benign tumor, with better understanding of dangerous anastomosis.
\end{abstract}

\section{Introduction}

Meningiomas are essentially benign tumors with symptoms developing in most cases due to compression by the tumor. Most patients rarely die due to this tumor. Conservative treatment is often selected for the asymptomatic cases. Thus, the primary goal of the surgeons is removal of the mass. Total excision of a skull base meningioma is accompanied by a risk because the surgical field is narrow, and the lesion is adjacent to nerves and blood vessels in many cases. The recurrence rate of meningioma varies among the Simpson grades of excision, and for a Simpson grade III or lower, excision is desirable. ${ }^{1}$ On the other hand, it has been found that the survival rate does not change with the excision rate. ${ }^{1}$

received

April 19, 2016

accepted after revision

December 1, 2016

published online

February 15, 2017

Thus, an in-depth investigation is necessary to decide on a treatment strategy.

\section{Subjects and Methods}

At our hospital, 78 meningioma patients were treated over the past 5 years, and 48 of these patients were treated with preoperative embolization. Skull base meningioma was found in 20 of the patients. Embolization was not used in the patients without feeders as determined by cerebral angiography. Embolization was usually performed under local anesthesia on the day before surgery. In all patients, a branch of the external carotid arterial system was occluded, and no embolization through the internal carotid (IC)

(c) 2017 Georg Thieme Verlag KG Stuttgart · New York
DOI https://doi.org/ 10.1055/s-0037-1598195. ISSN 2193-6331. 
artery was performed. Skull base meningioma was retrospectively investigated in the 20 patients with regard to the tumor location, maximum tumor diameter, occluded blood vessel, embolic materials, complications due to embolization, surgical time, bleeding time, and postsurgical neurologic findings.

\section{Results}

Clinical and surgical characteristics of all patients are presented in -Table 1. Microcatheter insertion into the feeding vessel arising from the occipital artery (OA) was impossible, and embolization was not performed in only one patient. In the other 19 patients, one to five feeding vessels could be embolized. N-butyl-2-cyanoacrylate (NBCA) was used in $80 \%$ of these patients. Only coils were used in the cases of frontal skull base meningioma in which the ethmoidal artery arising from the ophthalmic artery was the feeding vessel because the possibility of the liquid embolic agent entering the ophthalmic artery was of con- cern. Coils were usually placed proximal or distal to the blood vessel to be occluded to block misdirection of the liquid embolic agent, and the agent was infused after placing the coils. Particles were used only when a microcatheter could not be advanced sufficiently close to the tumor. To confirm the risk of neuropathy, lidocaine tests were applied to both the ascending pharyngeal artery (APA) and the posterior branch of the middle meningeal artery (MMA).

For normal meningioma, we found that preoperative embolization could be completed within approximately 1 hour under local anesthesia, and excision could be completed within 3 to 5 hours. However, a longer surgical time for excision and a larger loss of blood loss were recorded in cases of large and deeper tumors. Blood loss was normally approximately $250 \mathrm{~mL}$, but $800 \mathrm{~mL}$ were lost on average in four patients with the following conditions: one patient had a malignant meningioma, one patient had a large tumor located on the medial side of the sphenoidal ridge, and two had tumors that infiltrated the petroclival bone and the sigmoid

Table 1 Summary of embolization for skull base meningioma

\begin{tabular}{|c|c|c|c|c|c|c|c|c|c|c|}
\hline Location & Cases & $\begin{array}{l}\text { Age } \\
\text { and } \\
\text { sex }\end{array}$ & $\begin{array}{l}\text { Tumor } \\
\text { size } \\
(\mathrm{cm})\end{array}$ & $\begin{array}{l}\text { Feeding } \\
\text { arteries }\end{array}$ & Materials & $\begin{array}{l}\text { Number of } \\
\text { occluded } \\
\text { branch }\end{array}$ & $\begin{array}{l}\text { Simpson } \\
\text { grade }\end{array}$ & $\begin{array}{l}\text { Surgical } \\
\text { time(h) }\end{array}$ & $\begin{array}{l}\text { Bleeding } \\
(\mathrm{mL})\end{array}$ & Note \\
\hline \multirow{3}{*}{$\begin{array}{l}\text { Frontal } \\
\text { base }\end{array}$} & 1 & $73 \mathrm{M}$ & 4.7 & MMA & NBCA & 1 & 2 & 4.3 & 200 & \\
\hline & 2 & $67 \mathrm{~F}$ & 4 & MMA & $\begin{array}{l}\text { NBCA coil } \\
\text { particle }\end{array}$ & 2 & 2 & 4 & 150 & Lidocaine test \\
\hline & 3 & $81 \mathrm{M}$ & 50 & $\begin{array}{l}\text { Maxillary } \\
\text { artery }\end{array}$ & NBCA coil & 5 & 2 & 4.2 & 1,000 & Malignant meningioma \\
\hline \multirow[t]{6}{*}{$\begin{array}{l}\text { Sphenoidal } \\
\text { ridge }\end{array}$} & 4 & $54 \mathrm{M}$ & 80 & $\begin{array}{l}\text { MMA, } \\
\text { maxillary } \\
\text { artery }\end{array}$ & Particle & 3 & 2 & 6 & 320 & \\
\hline & 5 & $70 \mathrm{M}$ & 5.2 & $\begin{array}{l}\text { MMA, } \\
\text { maxillary } \\
\text { artery }\end{array}$ & NBCA coil & 4 & 2 & 5 & 310 & \\
\hline & 6 & $62 \mathrm{~F}$ & 5 & MMA & NBCA coil & 2 & 2 & 5 & 600 & $\begin{array}{l}\text { Visual deficit for } 6 \text { mo due } \\
\text { to embolization }\end{array}$ \\
\hline & 7 & $44 \mathrm{~F}$ & 4.3 & MMA & NBCA & 1 & 3 & 8 & 350 & \\
\hline & 8 & $69 \mathrm{~F}$ & 3 & MMA & NBCA & 2 & 2 & 4 & 120 & \\
\hline & 9 & $68 \mathrm{~F}$ & 5.5 & $\begin{array}{l}\text { Ethmoidal } \\
\text { artery }\end{array}$ & Coil & 1 & 4 & 3 & 200 & \\
\hline Petroclival & 10 & $50 \mathrm{M}$ & 4.2 & MMA & NBCA & 1 & 4 & 12 & 900 & $\begin{array}{l}\text { Gamma knife for } \\
\text { intracavernous tumor }\end{array}$ \\
\hline \multirow[t]{4}{*}{ CP angle } & 11 & $72 \mathrm{~F}$ & 6.2 & $\mathrm{OA}$ & NBCA & 2 & 3 & 4 & 160 & \\
\hline & 12 & $72 \mathrm{~F}$ & 5.3 & MMA & NBCA & 2 & 3 & 5 & 230 & \\
\hline & 13 & $39 \mathrm{~F}$ & 4 & APA & NBCA & 1 & 2 & 4 & 200 & Lidocaine test \\
\hline & 14 & $61 \mathrm{~F}$ & 3 & $\mathrm{OA}$ & NBCA & 1 & 2 & 3 & 250 & \\
\hline Tentorial & 15 & $67 \mathrm{~F}$ & 5.3 & $\mathrm{OA}$ & NBCA & 1 & 2 & 7 & 200 & \\
\hline \multirow{5}{*}{$\begin{array}{l}\text { Posterior } \\
\text { fossa }\end{array}$} & 16 & $76 \mathrm{~F}$ & 3 & MMA & NBCA & 2 & 3 & 4 & 100 & \\
\hline & 17 & $43 \mathrm{M}$ & 8 & $\begin{array}{l}\text { MMA, } \\
\text { maxillary } \\
\text { artery }\end{array}$ & Particle & 3 & 2 & 6 & 320 & \\
\hline & 18 & $51 \mathrm{~F}$ & 5.3 & MMA & NBCA & 1 & 2 & 5 & 700 & Attachment at the sigmoid sinus \\
\hline & 19 & $77 \mathrm{~F}$ & 5 & $\mathrm{OA}$ & No & 0 & 3 & 4 & 150 & Impossible to catheterize \\
\hline & 20 & $64 \mathrm{M}$ & 5 & OA & MBCA coil & 2 & 1 & 3 & 300 & \\
\hline
\end{tabular}

Abbreviations: APA, ascending pharyngeal artery; CP angle, cerebellar- pontine angle; F, female; M, male; MMA, middle meningeal artery; NBCA, n-butyl-2-cyanoacrylate; OA, occipital artery. 


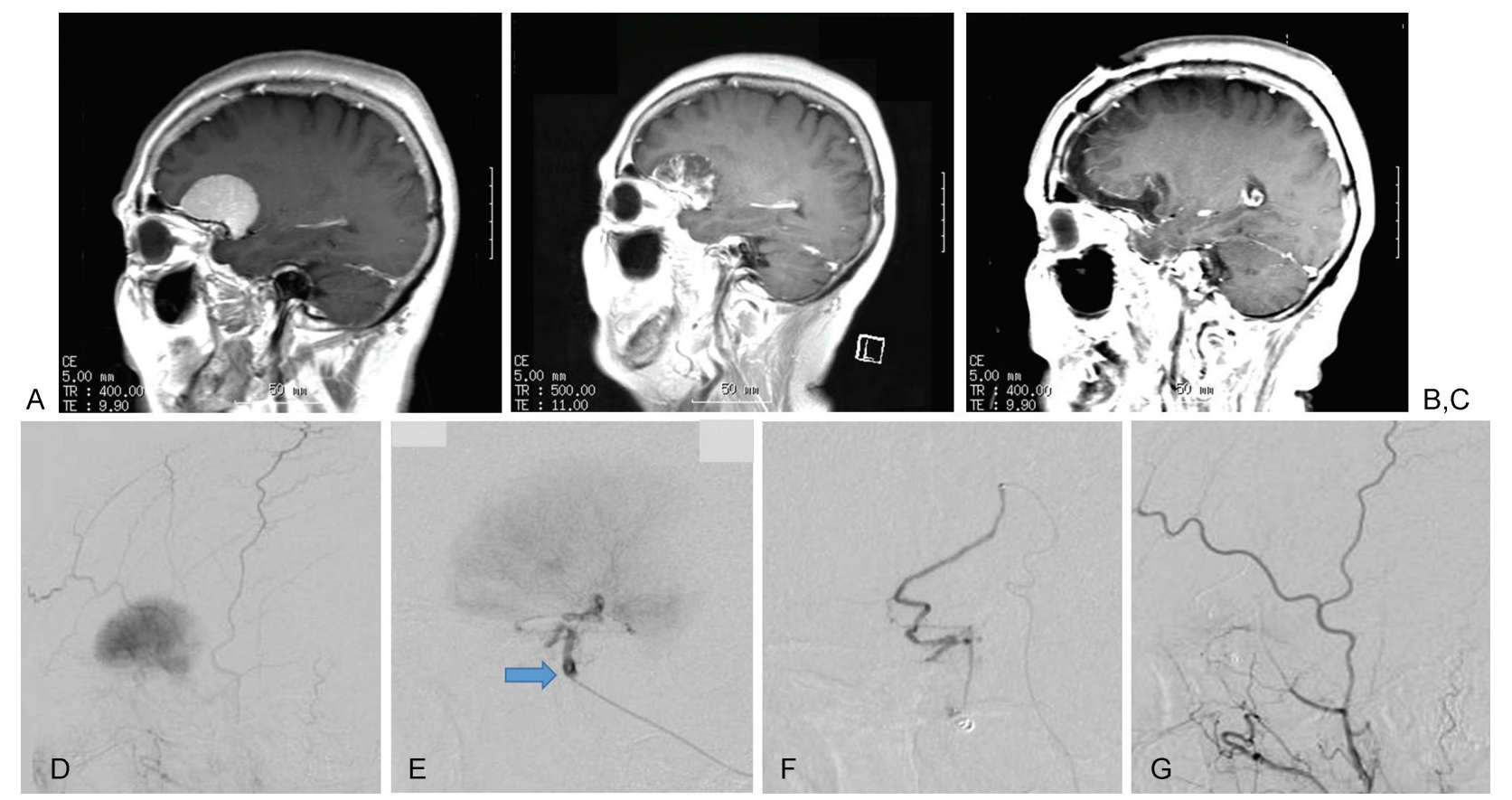

Fig. 1 Case 2: a 67-year-old female with a frontal base meningioma. (A) Pretreatment magnetic resonance imaging (MRI) showed a left frontal base meningioma with a diameter of $4 \mathrm{~cm}$. (B) Postembolization MRI showed a decreased area of the tumor. (C) Postsurgical MRI showed complete resection of the tumor. The Simpson grade was II. (D) A left external carotid arteriogram visualized the area of the tumor prior to treatment. (E) A microcatheter was inserted into a branch of the middle meningeal artery (MMA). We injected particles because of the distance to the intratumor vessel. After injection of the particles, we placed a coil at the arrow point. (F) A new microcatheter was place into another branch of the MMA. This branch was occluded by n-butyl-2-cyanoacrylate. (G) After embolization, the tumor was clearly decreased, with only attachment observable.

sinus. Ninety percent of the patients had a Simpson grade III excision, and $10 \%$ of the patients (two patients) had a Simpson grade IV excision. No permanent complications were caused by the preoperative embolization procedure, but the visual function of one patient was impaired for 6 months, and this problem was assumed to be due to unintended spread of the embolic material through the anastomosis between the recurrent MMA and ophthalmic artery formed due to MMA embolization. Fever developed after embolization in five patients, but it was not regarded as a complication. No neurologic problems were noted after the excision, and 19 patients achieved rehabilitation into society, excluding the patient with malignant meningioma.

\section{Case 2: A 67-Year-Old Woman}

A brain tumor was found in the left frontal skull base on close examination of headache (-Fig. 1A). Since intense staining from the MMA was noted on cerebral angiography (-Fig. 1D), embolization was performed on the day before tumor excision by craniotomy. A 6-Fr sheath was inserted through the right inguinal region under local anesthesia, and 6-Fr Roadmaster (Goodman) was inserted into the left external carotid artery. An Excelsior 1018 (Stryker Neurovascular) was inserted into the left MMA using Traxcess14 (Terumo) and advanced close to the tumor. At this point, the microcatheter could not be advanced any further, resulting in a slight gap to the tumor. Because there was concern about anastomosis formation between the recurrent MMA and the ophthalmic artery, $20 \mathrm{mg}$ of lidocaine was slowly infused to perform a provocation test. The result was negative, but no liquid embolic agent was used. Then, $10 \mathrm{~mL}$ of 10 -fold diluted 300- to 500- $\mu \mathrm{m}$ Embosphere (BioSphere Medical) were injected ( - Fig. 1E). At this point, blood flow from this blood vessel to the tumor was markedly reduced, but two sets of $E D$ coil Extrasoft (Kaneka) measuring $2 \times 3 \mathrm{~cm}$ were additionally placed to occlude the feeder to ensure embolization. Blood flow from another branch of the MMA remained, for which a 165-cm Marathon (Micro Therapeutics) was guided to the branch using a Tenrou1014 (Japan Lifeline). Considering that this branch has no risk of dangerous anastomosis, $0.1 \mathrm{~mL}$ of $17 \%$ NBCA was injected, and the intratumor embolization was successful (-Fig. 1F). On the final angiography, the tumor shadow was greatly reduced ( - Fig. 1G) with more than half of the tumor not able to be visualized with contrast medium on magnetic resonance imaging (MRI; - Fig. 1B), visually demonstrating the success of the embolization. Excision by frontotemporal craniotomy was performed on the following day. The dura of the frontal skull, which was the site of tumor attachment, was cauterized, and a Simpson grade II excision was performed (-Fig. 1C). The surgical time was 4 hours. No neurologic manifestations occurred after the surgery, and the patient was discharged.

\section{Case 6: A 62-Year-Old Woman}

A left meningioma was revealed by MRI as an examination of headache (-Fig. 2A). The tumor was in the middle third sphenoidal ridge and was fed by the right MMA ( - Fig. 2B). In the day before the surgery, preoperative embolization was 

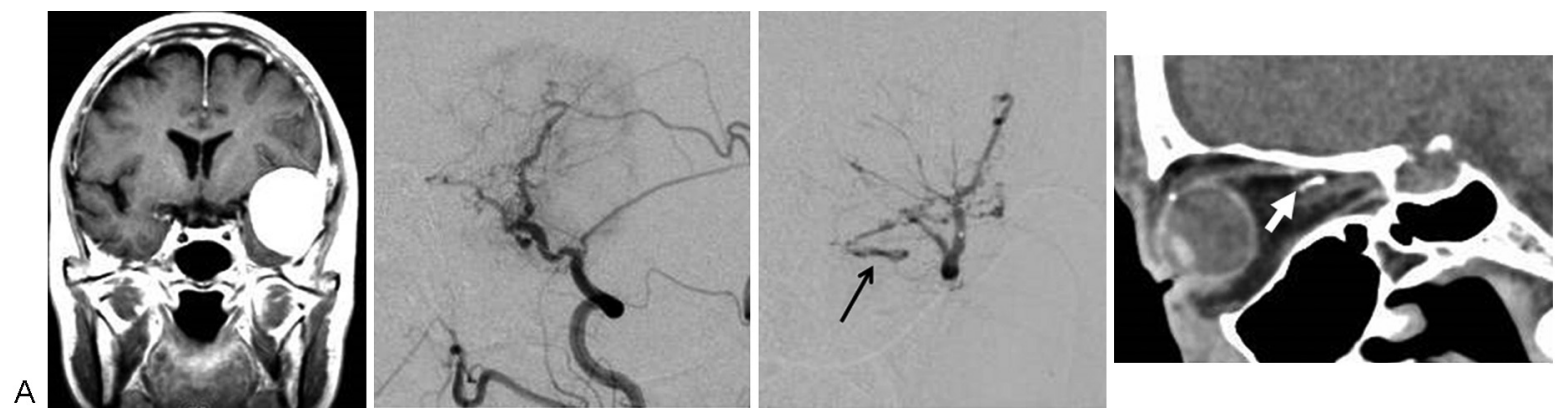

Fig. 2 Case 6: a 67-year-old woman with meningioma in the middle third of the sphenoidal ridge. (A) The maximum tumor diameter was diameter in $5 \mathrm{~cm}$. (B) Left external carotid angiogram presented tumor stain from middle meningeal artery (MMA). (C) N-butyl-2-cyanoacrylate (NBCA) was injected from the left MMA. Unknown brunch was presented (black allow). It might be the recurrent meningeal artery. (D) A high-density finding inside the orbit was presented in computed tomography (white allow). It was migration of NBCA into the ophthalmic artery.

performed. Under local anesthesia, a 6-Fr sheath was inserted, and a 90-cm 6-Fr Launcher (Medtronic Vascular) was placed in the left external carotid artery. A $165-\mathrm{cm}$ Marathon was guided to the MMA, and $0.2 \mathrm{~mL}$ of $20 \% \mathrm{NBCA}$

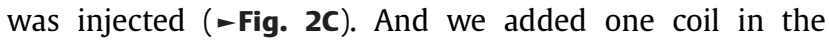
proximal of MMA. The patient had no symptom after the embolization. However, the next morning, she noticed disturbance of the left visual acuity. Emergent computed tomography revealed a high-density finding in the left orbit ( - Fig. 2D). We diagnosed that NBCA was migrated into the ophthalmic artery though the recurrent meningeal artery, which was known as one of the dangerous anastomosis. After the embolization, total removal (Simpson II) was performed as the schedule. Her visual acuity was gradually better and recovered 4 months later from the embolization.

\section{Case 13: A 39-Year-Old Woman}

The disease initially presented with right hearing impairment, and a tumor was found in the right cerebellopontine (CP) angle on MRI ( - Fig. 3A). On cerebral angiography, the tumor was found to be fed by the APA. Thus, embolization of the tumor blood vessel was performed on the day before excision. Under local anesthesia, a 6-Fr sheath was inserted through the right inguinal region, and a 90- $\mathrm{cm}$ 6-Fr Launcher was placed in the right external carotid artery. A 165-cm Marathon was guided to the APA using a Tenrou10 (Japan Lifeline) and into the tumor ( - Fig. 3B). Embolization from this region was considered safe, and $0.2 \mathrm{~mL}$ of $20 \%$ NBCA was injected. The shadow of the tumor completely disappeared. Tumor excision by craniotomy was performed on the following day. There was no bleeding from the inside of the tumor, and the tumor was very soft and could be easily excised ( - Fig. 3C). A Simpson grade II excision was possible ( - Fig. 3D), and surgery was completed within 4 hours. The hearing impairment improved after surgery, and the patient was discharged without any problems.

\section{Discussion}

This report presents the usefulness of preoperative embolization for skull base meningiomas. Embolization decreases bleeding and softens the tumor, and thus makes the surgery easier and safer to perform and reduces the surgical time. ${ }^{2}$ However, embolization is useless when the risk of the proce- dure exceeds its potential beneficial effect because the final objective is excision. Negative opinions on preoperative embolization are occasionally reported. ${ }^{3,4}$ An influence of preoperative embolization on the extent of the pathological problem was also reported, and attention should be paid to the potential for overgrading the pathology of the tumor. ${ }^{5}$ This technique has been performed since the $1980 \mathrm{~s}^{6}{ }^{6}$ and the current procedure is sophisticated and well tolerated. ${ }^{7}$

Cerebral endovascular treatment has markedly progressed over the last decade with the progression of improved devices. $^{7-9}$ As the indication expanded and the number of patients increased, the treatment became more sophisticated and the safety improved. For preoperative embolization, improvement in the microcatheter operability, development of the balloon catheter, and novel embolic materials have been very effective. It is essential to understand that an anastomosis of the external carotid artery system is very dangerous. The relationship between the MMA and either the ophthalmic artery or facial nerve, involvement of the APA, and anastomosis between the OA and vertebral artery (VA) are particularly important in defining the approach for the embolization (-Table 2).

Embolic materials, including coils, liquid embolic agents (NBCA, Onyx), and particles, are used alone or in combination depending on the different conditions. We attempted complete obstruction of the tumor blood vessel with a liquid embolic agent when a microcatheter was advanced sufficiently close to the tumor. When sufficient distal insertion was not possible, we used 300- to 500- $\mu \mathrm{m}$ Embosphere because even though the particles enter an important nerve-feeding blood vessel, particles of this size are less likely to cause neurologic manifestations. ${ }^{10}$ Particles even with a larger size may cause neurologic manifestations, and it has been reported that the risk of intratumor bleeding was lower than expected. ${ }^{11}$ The rate of embolization with liquid embolic agents is apparently higher, but as described previously, complications caused by preoperative embolization should be avoided. To prevent entrance of embolic materials into the normal structures, we also adopt a method in which the proximal or distal side of the blood vessels with a risk is blocked with coils and then either a liquid embolic agent or particles are injected only into the target blood vessel. When blood flow in the vessel can also occur through collateral 

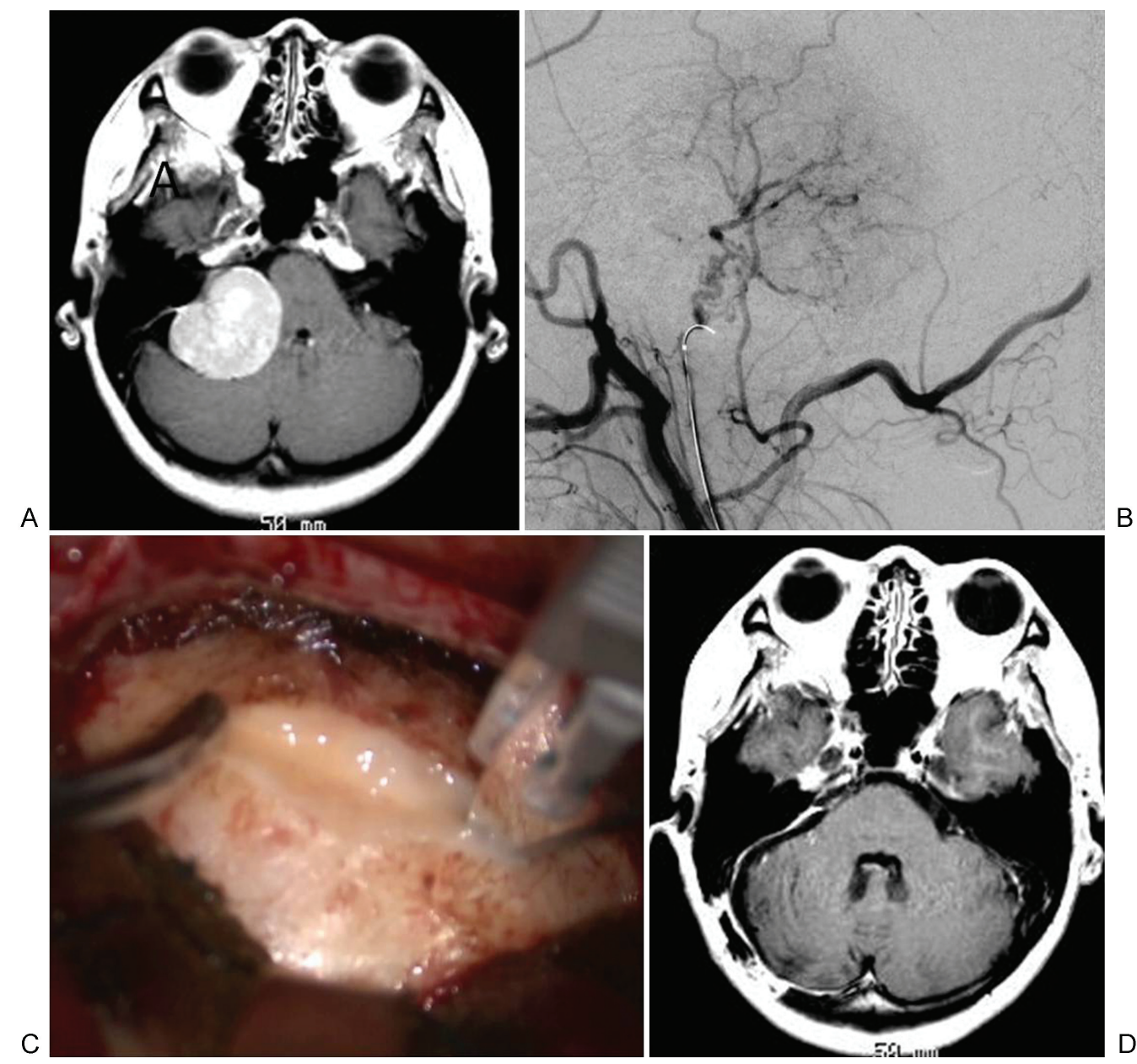

Fig. 3 Case 13: a 39-year-old female with cerebellopontine angle meningioma. (A) The maximum tumor diameter was $4 \mathrm{~cm}$. (B) The ascending pharyngeal artery was the feeding artery, and we embolized it with n-butyl-2-cyanoacrylate. (C) Surgical findings showed white and soft tumor due to the embolization. (D) Postoperative magnetic resonance imaging showing complete resection of the tumor. The Simpson grade was II.

circulation, embolization is safe. To prevent backflow of embolic materials, we also have adopted a method of injecting a liquid embolic agent, Onyx, through a double-lumen microcatheter (Scepter XC [Terumo]). Using this method, the embolic agent can be injected into a thin branch by blocking the original blood flow, enabling firm embolization, but the possibility of adherence of the catheter should be taken into consideration. Moreover, embolization of the feeding artery arising from the IC artery is controversial. A method of embolization that avoids unintended entrance of embolic material into the IC artery by blocking the peripheral side of the IC artery with a balloon catheter has been reported. Embolization should be planned taking the risks and benefits into consideration. ${ }^{8,12,13}$

For embolization of the MMA, which is relatively frequently performed, anastomosis of the recurrent MMA with the ophthalmic artery is important for determining the method of embolization. ${ }^{14}$ If a catheter can be advanced sufficiently distal to the frontal base of the skull in the lateral view, complete liquid embolization is possible, but when it is not

Table 2 Dangerous anastomosis from the external carotid artery

\begin{tabular}{|l|l|l|}
\hline External carotid artery & Branches & Terminal \\
\hline Middle meningeal artery(MMA) & Recurrent meningeal artery & Ophthalmic artery \\
\cline { 2 - 3 } & Posterior branch & VII \\
\hline Ascending pharyngeal artery (APA) & Neuromeningeal branch & VI, IX, X, XI, XII \\
\cline { 2 - 3 } & - & Vertebral artery \\
\cline { 2 - 3 } & - & Internal carotid artery \\
\hline Occipital artery & - & Vertebral artery \\
\hline
\end{tabular}

Roman numerals mean the cranial nerves. 
possible, embolization with particles is safe. The visual function of one of our patients was disturbed after embolization of the MMA with NBCA. Based on this experience with the MMA embolization, we recommend that injection should be immediately discontinued when contrast medium enters toward the orbit in the frontal and lateral views. Since embolization of the posterior branch of the MMA may affect the facial nerve, attention should be paid to this possible complication, which may be a good indication for doing the lidocaine test. ${ }^{15}$

The APA may be involved in meningioma of the $\mathrm{CP}$ angle. The APA nourishes cranial nerves VI, IX, X, XI, and XII, and anastomosis of the APA with both the VA and IC artery has been reported. The neuromeningeal trunk is a particularly dangerous branch, but we judge embolization as safe when a microcatheter is inserted and only the tumor vessel is enhanced with the dye. The provocation test using lidocaine as described previously is effective and is performed because involvement of the posterior branch of the MMA and APA in the cranial nerves has also been reported. ${ }^{15}$ We perform embolization with either particles or NBCA only when the provocation test is negative.

When embolization is successful, bleeding obviously decreases. $^{2}$ When no bleeding occurs, and the tumor is softer, the surgery is much easier to perform. Dissection from the surrounding normal tissue becomes less complicated, and the procedure is performed in a visually dry field, thus improving the safety of the procedure. ${ }^{16}$ The surgical time is shortened, which is particularly advantageous for the elderly. If no complications occur, the efficacy of embolization is apparent. Thus, the frequency of complications is important. The incidence of complications caused by preoperative embolization for meningioma has been described in many reports. ${ }^{2-4,7-12,16,17}$ The Neurosurgical Focus in 2013 reported that the risk was previously $21 \%$ but it had recently decreased to $6 \%,{ }^{7}$ and it further decreased to $1.0 \%$ in $2015 .^{8}$ The only complication in our study other than fever was a visual disturbance that persisted for 6 months in $1(1.5 \%)$ out of all 65 patients treated with meningioma embolization.

Embolization results have been improving each year because of the increasingly sophisticated cerebral endovascular techniques. Safe and reliable excision of skull base meningioma, which is very difficult, is a major objective of neurosurgeons. In surgery for skull base meningioma, the feeding vessel is processed in the latter half of the surgery, for which preoperative embolization is very beneficial. ${ }^{9}$ For convexity meningioma of the posterior skull, the feeding vessel can be processed at the beginning of the surgery, for which preoperative embolization is not necessarily indicated. We did not perform a preoperative embolization in a patient with this type of meningioma because guiding the microcatheter was difficult. Since the same team performs both preoperative embolization and excision, treatment can be performed while considering the risks of intraoperative embolization and excision. It is necessary to decide on the indication of preoperative embolization in consideration of what is the best for the patients. When treatment is performed by two teams, it is essential to prepare a treatment strategy by closely exchanging information between the endovascular and surgical teams.

\section{Limitations}

This was a retrospective study performed at a single institution involving a small number of patients, and thus the amount of data was limited. Since surgery of the skull base requires advanced techniques, experienced surgeons should be selected. Some researchers consider that the efficacy of preoperative embolization of meningioma is not evaluable because there are insufficient data, ${ }^{7}$ and it may be very difficult to evaluate such challenging skull base surgery based on evidence. We cannot compare directly between with and without preoperative embolization in this study.

\section{Conclusion}

The efficacy of preoperative embolization for skull base meningioma was reported. Preoperative embolization makes the surgery easier. The risk of preoperative embolization has decreased considerably and continues to decrease each year, and it is now within the acceptable range. However, treatment should be performed while considering latent risks, including a dangerous anastomosis, and treatment performed by one team is advantaged and preferable.

\section{References}

1 Gousias K, Schramm J, Simon M. The Simpson grading revisited: aggressive surgery and its place in modern meningioma management. J Neurosurg 2016;125(3):551-560

2 Ishihara $\mathrm{H}$, Ishihara S, Niimi J, et al. The safety and efficacy of preoperative embolization of meningioma with $\mathrm{N}$-butyl cyanoacrylate. Interv Neuroradiol 2015;21(5):624-630

3 Rosen CL, Ammerman JM, Sekhar LN, Bank WO. Outcome analysis of preoperative embolization in cranial base surgery. Acta Neurochir (Wien) 2002;144(11):1157-1164

4 Shah AH, Patel N, Raper DM, et al. The role of preoperative embolization for intracranial meningiomas. J Neurosurg 2013; 119(2):364-372

5 Barresi V, Branca G, Granata F, Alafaci C, Caffo M, Tuccari G. Embolized meningiomas: risk of overgrading and neo-angiogenesis. J Neurooncol 2013;113(2):207-219

6 Manelfe C, Lasjaunias P, Ruscalleda J. Preoperative embolization of intracranial meningiomas. AJNR Am J Neuroradiol 1986;7(5): 963-972

7 Singla A, Deshaies EM, Melnyk V, et al. Controversies in the role of preoperative embolization in meningioma management. Neurosurg Focus 2013;35(6):E17

8 Aihara M, Naito I, Shimizu T, et al. Preoperative embolization of intracranial meningiomas using n-butyl cyanoacrylate. Neuroradiology 2015;57(7):713-719

9 Raper DM, Starke RM, Henderson F Jr, et al. Preoperative embolization of intracranial meningiomas: efficacy, technical considerations, and complications. AJNR Am J Neuroradiol 2014;35(9): 1798-1804

10 Dowd CF, Halbach VV, Higashida RT. Meningiomas: the role of preoperative angiography and embolization. Neurosurg Focus 2003;15(1):E10 
11 Law-ye B, Clarençon F, Sourour NA, et al. Risks of presurgical embolization of feeding arteries in 137 intracranial meningeal tumors. Acta Neurochir (Wien) 2013;155(4):707-714

12 Abdel Kerim A, Bonneville F, Jean B, Cornu P, LeJean L, Chiras J. Balloon-assisted embolization of skull base meningioma with liquid embolic agent. J Neurosurg 2010;112(1):70-72

13 Waldron JS, Sughrue ME, Hetts SW, et al. Embolization of skull base meningiomas and feeding vessels arising from the internal carotid circulation. Neurosurgery 2011;68(1):162-169, discussion 169

14 Hayashi N, Kubo M, Tsuboi Y, et al. Impact of anomalous origin of the ophthalmic artery from the middle meningeal artery on selection of surgical approach to skull base meningioma. Surg Neurol 2007;68(5):568-571, discussion 571-572

15 Kai Y, Hamada J, Morioka M, et al. Preoperative cellulose porous beads for therapeutic embolization of meningioma: provocation test and technical considerations. Neuroradiology 2007;49(5):437-443

16 Kominami S, Watanabe A, Suzuki M, Mizunari T, Kobayashi S, Teramoto A. Preoperative embolization of meningiomas with Nbutyl cyanoacrylate. Interv Neuroradiol 2012;18(2):133-139

17 Borg A, Ekanayake J, Mair R, et al. Preoperative particle and glue embolization of meningiomas: indications, results and lessons learned from 117 consecutive patients. Neurosurgery 2013. doi: 10.1227/NEU.0000000000000187 